\title{
Norois
}

Environnement, aménagement, société

$230 \mid 2014$

Histoire de la géographie, mobilités étudiantes, circuits courts, maillage territorial, régénération urbaine, hydrographie

\section{Circuits courts agroalimentaires de proximité en Limousin : performance économique et processus de gentrification rurale}

Rural Gentrification and economic performance of local short food supply chain in Limousin

Frédéric Richard, Marius Chevallier, Julien Dellier et Vincent Lagarde

\section{(2) OpenEdition}

Journals

Édition électronique

URL : https://journals.openedition.org/norois/4997

DOI : $10.4000 /$ norois.4997

ISBN : 978-2-7535-3509-1

ISSN : $1760-8546$

Éditeur

Presses universitaires de Rennes

Édition imprimée

Date de publication : 30 juin 2014

Pagination : 21-39

ISBN : 978-2-7535-3464-3

ISSN : 0029-182X

Référence électronique

Frédéric Richard, Marius Chevallier, Julien Dellier et Vincent Lagarde, « Circuits courts

agroalimentaires de proximité en Limousin : performance économique et processus de gentrification rurale », Norois [En ligne], 230 | 2014, mis en ligne le 30 juin 2016, consulté le 13 janvier 2022. URL : http://journals.openedition.org/norois/4997 ; DOI : https://doi.org/10.4000/norois.4997 


\title{
Circuits courts agroalimentaires de proximité en Limousin : performance économique et processus de gentrification rurale
}

\author{
Rural Gentrification and Economic Performance \\ of Local Short Food Supply Chain in Limousin
}

Frédéric Richard ${ }^{* a}$, Marius Chevallier ${ }^{\mathrm{a}}$, Julien Dellier ${ }^{\mathrm{a}}$ et Vincent Lagarde ${ }^{\mathrm{b}}$

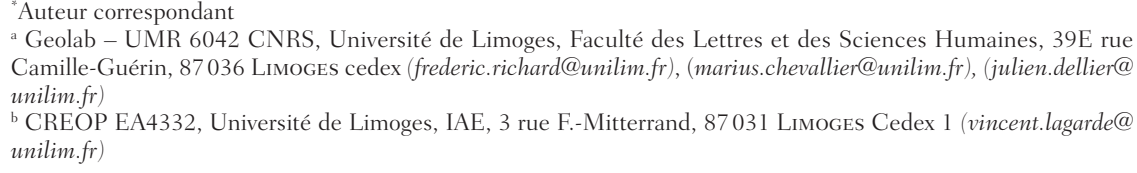

Résumé : En 2011, la Direction Régionale Agriculture Alimentation Forêt du Limousin et le Conseil Régional Limousin ont mis en place une enquête complémentaire au Recensement Agricole auprès de 507 des 1422 producteurs pratiquant les circuits courts de proximité (CCP) dans la région. Les données économiques, comparées à celles du Réseau d'Information Comptable Agricole, montrent que les exploitations pratiquant les CCP sont plus performantes que celles ne les pratiquant pas. Dans une perspective inductive, l'analyse économique et financière de ce différentiel de performance est complétée d'observations relatives aux dynamiques sociales et géographiques des CCP en Limousin. Il en ressort que l'appartenance des consommateurs et producteurs à une même classe sociale y favoriserait ainsi le développement et la performance des CCP. Le phénomène des CCP peut alors apparaître comme une manifestation du processus de gentrification rurale.

Abstract: In 2011, the DRAAF and Region Council of Limousin conducted an additional survey to the Agricultural Census with 507 of the 1422 producers involved in the short food supply chain (SFSC) in the region. The economic data, compared with the ones of the Farm Accountancy Data Network (FADN), show that the farms using local networks are more successful than those not using them. Developing an inductive approach, the economic and financial analysis of this performance gap is completed by empirical material related to the social and geographical dynamics of the SFSC networks in Limousin. It shows that the development and the performance of the SFSC networks would be favoured when local producers and consumers belong to the social class. These networks could therefore appear as an expression of the rural gentrification process.

Mots clés : performance économique - circuits courts de proximité - agriculture - recomposition sociale - gentrification rurale Keywords: economic performance - short food supply chain-agriculture - social change - rural gentrification 


\section{INTRODUCTION}

Les circuits courts de proximité $(\mathrm{CCP})^{1}$, forme traditionnelle mais progressivement marginalisée de commercialisation dans le domaine agroalimentaire, sont actuellement réinvestis en réponse aux critiques du modèle agro-industriel dominant (Goodman et Watts, 1997 ; Van der Ploeg et al., 2000; Lamine et Perrot, 2008). Ils sont ainsi censés contribuer aux trois piliers du développement durable (Transrural Initiatives, 2006, 2007). D'un point de vue environnemental, ils favoriseraient les pratiques culturales " raisonnées » et seraient plus économes en gaz à effet de serre que les circuits conventionnels. D'un point de vue économique, la réduction du nombre d'intermédiaires permettrait aux agriculteurs de reprendre le contrôle de la valeur ajoutée et d'améliorer leurs revenus. Enfin, en termes sociaux, en rendant plus accessibles des produits frais de qualité à un éventail toujours plus large de consommateurs, les CCP rapprocheraient les deux extrémités de la filière : ils reconnecteraient, voire réconcilieraient, producteurs et consommateurs, seraient un moyen de renouer les échanges entre ruraux et citadins et de rééquilibrer les rapports ville-campagne.

Nous proposons de réinterroger ici ces deux derniers aspects, c'est-à-dire les revenus des producteurs et le rôle des échanges sociaux et ce, à partir d'une analyse de cas portant sur la région Limousin. L'hypothèse selon laquelle les CCP permettent d'améliorer les revenus des agriculteurs a été peu étudiée, ou plutôt peu approfondie. Pourtant, cet argument est suffisamment admis et partagé pour être à l'origine de politiques publiques ou d'initiatives diverses en matière d'accompagnement des agriculteurs intéressés par ce mode de commercialisation; il est en particulier avancé comme une réponse pertinente aux difficultés plus ou moins conjoncturelles des producteurs. En Limousin par exemple, région aux résultats des exploitations agricoles les plus faibles de France (Allirot et al., 2012), l'accélération des dépôts de bilan au début des années 2000

1. La notion de « circuit court » a été définie dans le Plan Barnier en 2009 par le nombre d'intermédiaires (un maximum) entre le producteur et le consommateur quelle que soit la distance kilométrique. En Limousin, par ordre d'importance, les modes de vente sont : à la ferme, commerçants, marchés, tournées alimentaires, points de vente collectifs, restauration commerciale, grandes et moyennes surfaces, salons, correspondance, paniers, restauration collective. La notion de «circuit de proximité » fait référence au nombre de kilomètres entre le lieu de production et le lieu de consommation quels que soient le nombre d'intermédiaires. La présente étude s'intéresse aux circuits qui sont à la fois courts et de proximité. a incité les services déconcentrés de l'État (Draaf) et la Région à lancer un programme de recherche soutenu financièrement par le Ministère de l'agriculture et scientifiquement par l'UMR Geolab 6042 CNRS de l'Université de Limoges. Globalement, les objectifs de la recherche consistaient dans un premier temps à mieux connaître les producteurs recourant aux CCP, les dynamiques (notamment sectorielles et géographiques) et conditions d'installation, de diversification et de développement de ces «nouvelles » formes de commercialisation pour, in fine, se doter des meilleurs outils pour encourager et soutenir efficacement leur développement.

Paradoxalement, ce n'est qu'a posteriori, grâce aux données issues de l'étude (cf. infra), combinées à celles du Recensement Agricole 2010, que nous sommes en mesure de valider le postulat de départ d'une meilleure performance économique des exploitations commercialisant en CCP, constituant par ailleurs un apport empirique relativement inédit au regard du corpus et de la méthodologie retenue. Au-delà de ce résultat, l'étape suivante a consisté à déterminer les facteurs explicatifs de ce différentiel de performance. Malheureusement, pour volumineux qu'il soit, le corpus de données disponible a trop rapidement imposé les limites d'une analyse de type économique ou comptable. Mais de fait, les circuits-courts constituent un objet protéiforme qui, par nature, devrait donner lieu à des recherches pluridisciplinaires. En l'occurrence, les chercheurs engagés dans ce programme ont eu l'opportunité de s'appuyer sur d'autres travaux conduits en Limousin pour envisager une lecture interprétative autre qu'économique pour tenter d'expliquer le développement et la bonne performance des CCP dans la région. Cette démarche strictement inductive nous a ainsi conduits à analyser la performance économique des CCP au travers de leur articulation avec l'ensemble des mutations fonctionnelles, sociales et géographiques qui affectent les espaces ruraux et plus explicitement avec la gentrification des campagnes françaises, ou a minima, de certaines d'entre elles.

Concrètement, les CCP ne sont pas en « lévitation », ils sont au contraire inscrits, voire encastrés (Hinrichs, 2000; Winter, 2003), au sein de territoires d'échelles variables et de structures sociales localisées ou de sociétés locales dont ils sont issus et qu'ils contribuent à produire. Parmi les trans- 
formations des espaces ruraux, le renouvellement des populations, principalement sous l'effet des dynamiques migratoires, constitue un fait majeur. Ces mouvements reflètent et s'accompagnent de mutations fonctionnelles : d'espaces productifs, les espaces ruraux deviendraient peu à peu " postproductifs »(Marsden et al., 1993), à vocation essentiellement résidentielle et sont même parfois présentés comme des espaces « consommés ». Ces mutations seraient en particulier le résultat des rapports qu'entretiennent les nouvelles populations résidentes avec les campagnes, nouvelles populations dont le profil socio-économique et culturel est souvent fondamentalement différent de celui des populations dites locales ou autochtones ${ }^{2}$. Ce remplacement progressif des populations aux capitaux économiques et/ou culturels en moyenne plus modestes que ceux des nouveaux arrivants, a poussé un certain nombre d'auteurs à mobiliser le concept de gentrification rurale pour interpréter l'évolution des campagnes (Phillips, 1993), y compris françaises (Raymond, 2005; Pistre, 2012). En résumé, l'objectif serait ici de réfléchir et de poser les jalons de recherches ultérieures quant aux liens susceptibles d'être tissés entre, d'une part le processus de gentrification rurale, et d'autre part, les CCP agroalimentaires, leurs modalités de développement ainsi que leur efficacité économique.

Dans cette perspective, nous avons fait le choix d'organiser cette contribution à l'image du cheminement intellectuel qui nous a progressivement amenés de la déconstruction statistique et comptable de la performance économique des exploitations installées en CCP, à l'analyse des composantes sociales et économiques de la gentrification rurale et de l'influence de celle-ci sur le développement des circuits-courts en Limousin.

\section{UNE MEILLEURE PERFORMANCE ÉCONOMIQUE DES EXPLOITATIONS PRATIQUANT LES CCP}

\section{La faible connaissance des revenus des agriculteurs pratiquant les CCP}

Logiquement, du fait de la réduction, voire de la suppression, des intermédiaires, la meilleure capta-

2. Avec toute la prudence et réserves susceptibles de s'appliquer à la notion (voir par exemple Girard A., 2012). tion de la valeur ajoutée par les exploitations devrait leur être profitable en termes de performance économique et de revenus. Paradoxalement, comme le soulignent encore récemment les auteurs du rapport Short Food Supply Chains and Local Food Systems in the EU. A State of Play of their Socio-Economic Characteristics, si les assertions allant dans ce sens sont nombreuses, très peu sont étayées par des recherches empiriques. Lorsque celles-ci existent, « the majority of evidence is qualitative, and based on perceptions and experiences ${ }^{3}$ » (Santini, Gomez y Paloma, 2013), la plupart du temps, à partir d'études de cas locales. Par exemple, les analyses questionnent souvent la place des revenus parmi l'ensemble des motivations des agriculteurs ayant fait le choix de recourir à la vente directe (Cartier, 1994 ; Govindasamy et Nayga, 1996).

Quant aux recherches plus quantitatives ayant effectivement porté sur les résultats du changement de mode de commercialisation, elles s'appuient essentiellement sur l'évolution du chiffre d'affaires et n'autorisent que peu de comparaisons. Par exemple, sur les 557 exploitations enquêtées dans le New Jersey en 1992, celles qui pratiquent l'agrotourisme ou la vente directe dégagent un chiffre d'affaire par hectare plus élevé que les autres (Govindasamy et al., 1999). Aux États-Unis toujours, ces éléments ont été confortés par les données d'une enquête agricole annuelle publique nationale (ARMS : Agricultural Resource Management Survey) conduite en 2002 et doublée d'une enquête complémentaire en agriculture biologique. Ainsi, sur une population de 339 exploitations en agriculture biologique (soit $3 \%$ d'un échantillon de 13303 exploitations), celles qui recourent à la vente directe présentent un chiffre d'affaires supérieur de $3,4 \%$ à celles qui leur préfèrent les filières longues. Pour autant, faute d'information sur les charges, ces résultats ne peuvent être transposés en termes de revenu final (Detre et al., 2011). En France, une vaste étude relative à " l'Élaboration d'un référentiel technico-économique dans le domaine des CCP de commercialisation " (Capt et al., 2011) a permis de rassembler les données comptables détaillées d'un échantillon de quatre-vingt exploitations en maraîchage et quatre-vingt exploitations spécialisées en bovin lait localisées dans diverses régions

3. «La plupart des données sont qualitatives, basées sur les perceptions et expériences des personnes interrogées » 
de l'hexagone et toutes installées en circuits courts. Les auteurs ont ainsi pu élaborer une comptabilité analytique permettant d'isoler les activités circuits courts du reste des activités des exploitations, mais là-encore sans être en mesure d'engager de comparaison avec les exploitations en filières longues.

Au total, il semble que l'invitation de Hennebery et al. (2009) à travailler à l'évaluation précise du chiffre d'affaires des exploitations afin de mesurer les impacts économiques des circuits courts garde à ce jour toute sa pertinence.

\section{Des revenus supérieurs pour les exploitations en CCP en Limousin}

En l'occurrence, les données produites en Limousin nous offrent cette double opportunité de travailler sur les revenus finaux des exploitations en $\mathrm{CCP}$ et de comparer les revenus des exploitations en filières courtes et longues. L'enquête dénommée Circuits Courts en Limousin (CCL ci-après) a été réalisée en 2011 par le Conseil Régional du Limousin et la Draaf Limousin ${ }^{4}$, avec la participation de Geolab. Menée par dix enquêteurs de la Draaf, elle offre un taux de sondage et une représentativité probablement inédits pour un territoire de cette échelle puisque 507 des 1422 producteurs identifiés comme pratiquant les circuits courts 5 à l'occasion du Recensement Agricole de 2010 ont pu être enquêtés (Allirot et al., 2012; Chevallier 2013a; Chevallier 2013b; Chevallier et al., à paraître). En complément de ce corpus quantitatif, une enquête qualitative s'est concrétisée par une quarantaine d'entretiens semi-directifs conduits auprès de consommateurs, producteurs, commerçants et institutionnels. Tous engagés dans une démarche de CCP, ils ont été sélectionnés de manière à constituer un échantillon représentatif des acteurs et territoires limousins en fonction de critères de production (sept filières), modes de commercialisation (cinq modes) et de localisation (six zones géographiques en Limousin). Quoi qu'il en soit, parmi les 700 variables renseignées dans

4. Service Régional de L'information Statistique, Economique et Territoriale (SRISET), que nous remercions pour l'accès aux données et la contribution à l'analyse financière.

5. Il s'avère en fait qu'en Limousin les circuits courts sont quasi exclusivement de proximité comme en témoigne l'analyse des lieux de vente cités et des kilomètres parcourus pour la commercialisation. Il est donc légitime a posteriori de parler de CCP. l'enquête CCL, trois nous intéressent plus particulièrement ici : le chiffre d'affaires, les charges et les annuités bancaires. Par ailleurs, le profil des exploitations enquêtées, notamment en matière de surface agricole utile (SAU), unités de travail annuel (UTA) et effectifs animaux, a pu être directement complété à partir du Recensement Agricole.

Or, tous ces éléments comptables et structurels ont la particularité d'être également disponibles au travers du Réseau d'Information Comptable Agricole (RICA), rendant ainsi possible une démarche comparative significative entre d'un côté l'ensemble des exploitations ${ }^{6}$, et de l'autre les seules exploitations installées en CCP. Les résultats de cette comparaison sont donnés pour les moyennes et grandes exploitations spécialisées en bovin viande. En effet, d'une part le RICA ne concerne que les moyennes et grandes exploitations ${ }^{7}$. D'autre part, seules quatre productions présentent un nombre significatif d'exploitations agricoles enquêtées dans les deux enquêtes CCL et RICA pour autoriser des comparaisons (figure 1), les données n'étant suffisamment robustes que pour le bovin viande ${ }^{8}$. Cette double contrainte impose donc de réduire le champ d'analyse qui reste toutefois pertinent : non seulement les moyennes et grandes exploitations représentent $59 \%$ des exploitations qui pratiquent la vente en CCP (avec 10,6\% d'entre elles qui commercialisent tout ou partie de leur production contre $8,6 \%$ pour les petites) mais en outre, le bovin viande constitue de loin la principale production en Limousin. Précisons par ailleurs que les données comparées sous-estiment les écarts réels entre les exploitations pratiquant les CCP et les autres. En effet, il n'a pas été possible d'exclure de l'échantillon RICA les exploitations pratiquant les CCP : la comparaison se fait donc entre des exploitations pratiquant les CCP

6. Le différentiel de performance mesuré en faveur des exploitations en CCP est probablement supérieur dans les faits. En effet, l'échantillon RICA comprend des exploitations à la fois en filières courtes et longues. Or, pour mesurer le différentiel de performance, il faudrait idéalement en exclure les exploitations en CCP. Cela aurait nécessité un appariement avec les données individuelles du RA auxquelles nous n'avons pas accès.

7. Cela ne signifie pas que nous reprenions à notre compte l'idée sousjacente à la construction du RICA en 1965 que seules les moyennes et grandes exploitations seraient «professionnelles » : « L'enquête ne couvre toutefois pas l'ensemble des exploitations agricoles de l'Union mais uniquement celles ayant une dimension suffisante pour pouvoir être considérées comme professionnelles » ([http://ec.europa.eu/agriculture/rical concept_fr.cfm], consulté le 21 janvier 2014).

8. Les données en ovin viande, pomiculture et bovin lait ne sont pas robustes statistiquement mais les résultats vont dans le même sens que celles pour le bovin viande (Chevallier, 2013b). 
Les 220 exploitations du RICA et les 507 exploitations de $C C L$ ne peuvent être comparées directement. Tout d'abord, le RICA ne porte que sur les exploitations moyennes et grandes. Ensuite, les orientations productives ayant un impact fort sur les équilibres économiques, il est nécessaire de réaliser des comparaisons intra-sectorielles. La nomenclature des OTEX (orientations technico-économiques) permet de repérer 4 productions (3900, arboriculture ; 4500, bovin lait ; 4600, bovin viande ; 4813, ovins-caprins) dans lesquelles les exploitations enquêtées sont suffisamment nombreuses dans les deux bases de données. Plus précisément, on constate que les exploitations de la base RICA des OTEX 3900 et 4813 sont respectivement exclusivement en pomiculture et ovin viande. Les exploitations de l'enquête CCL spécialisées en petits fruits sont donc exclues de la comparaison, de même que les exploitations caprines. Au final, on obtient deux échantillons comparables de 189 exploitations dans la base RICA et 152 dans la base CCL (tableau $A$ ).

\begin{tabular}{|l|r|r|}
\hline Effectifs enquêtés & RICA & \multicolumn{1}{c|}{ CCL } \\
\hline Fruits à pépins & 19 & 17 \\
\hline Bovin lait & 13 & 15 \\
\hline Bovin viande & 132 & 105 \\
\hline Ovin viande & 25 & 15 \\
\hline Total & 189 & 152 \\
\hline
\end{tabular}

Tableau A : exploitations enquêtées par orientation Oproductive. Sources : RICA 2010, Enquête CCL 2011

Cependant, dans l'enquête $\mathrm{CCL}$, les données économiques sont inégalement renseignées, de sorte que pour le revenu disponible, les effectifs peuvent descendre jusqu'à 8 exploitations en pomiculture, 8 en bovin lait et 7 en ovin viande. L'essentiel du travail de comparaison porte donc sur les exploitations spécialisées en bovin viande, système dominant en Limousin.
Pour ce qui est de la représentativité de ce nouvel échantillon pour chacune des bases de données, on vérifie que les moyennes des variables SAU et UTA du nouvel échantillon ne sont pas trop éloignées des moyennes de la population totale renseignées par les données du Recensement Agricole, sans qu'il ait néanmoins été possible de calculer des tests de différence de la moyenne, n'ayant eu accès qu'aux données individuelles CCL et RICA, mais pas aux données individuelles du Recensement Agricole. Pour les données $\mathrm{CCL}$, on constate que le taux de sondage est très élevé et que l'échantillon est fortement représentatif à l'aune des UTA, mais sur-représente légèrement les exploitations de SAU importante, notamment en pomiculture et en bovin lait (tableau B). Pour les données RICA (tableau C), la représentativité est de moins bonne qualité, toutefois, elle est correcte pour les exploitations en bovin viande qui constituent le cœur du travail de comparaison.

\begin{tabular}{|l|r|r|r|}
\hline \multicolumn{2}{|c|}{$\begin{array}{c}\text { moyennes et grandes } \\
\text { exploitations pratiquant } \\
\text { les circuits courts }\end{array}$} & \multicolumn{2}{c|}{ rapport des médianes } \\
\hline OTEX & tx de sondage & SAU_CC/SAU_RA & UTA_CC/UTA_RA \\
\hline 3900 & $37 \%$ & 1,13 & 0,94 \\
\hline 4500 & $36 \%$ & 1,13 & 0,99 \\
\hline 4600 & $43 \%$ & 1,08 & 1,00 \\
\hline 4813 & $47 \%$ & 1,00 & 1,00 \\
\hline
\end{tabular}

Tableau B - Vérification de la représentativité par OTEX de l'échantillon CCL

\begin{tabular}{|l|r|r|r|}
\hline \multicolumn{2}{|c|}{$\begin{array}{c}\text { moyennes et grandes } \\
\text { exploitations tous modes } \\
\text { de vente confondus }\end{array}$} & \multicolumn{2}{|c|}{ rapport des médianes } \\
\hline OTEX & tx de sondage & SAU_RICA/SAU_RA & UTA_RICA/UTA_RA \\
\hline 3900 & $8 \%$ & 1,37 & 1,61 \\
\hline 4500 & $3 \%$ & 0,91 & 1,21 \\
\hline 4600 & $3 \%$ & 0,98 & 1,08 \\
\hline 4813 & $4 \%$ & 0,83 & 1,21 \\
\hline
\end{tabular}

Tableau C - Vérification de la représentativité par OTEX de l'échantillon RICA

Richard F., Chevallier M., Dellier J., Lagarde V., 2014 Conception et réalisation : Geolab UMR 6042 CNRS - Université de Limoges

Figure 1 : Éléments de comparaison entre RICA et CCL Comparisons between RICA and CCL

(CCL) et un ensemble d'exploitations tous modes de commercialisation confondus 9 .

Au final, les principaux résultats issus des recoupements des deux bases de données sont synthéti-

9. Dont on peut faire l'hypothèse qu'elles constituent $10,6 \%$ de l'échantillon si l'échantillon RICA est représentatif de l'ensemble des moyennes et grandes exploitations sés à travers le tableau 1. Les chiffres de la partie supérieure du tableau confirment l'hypothèse de la meilleure performance des exploitations écoulant tout ou partie de leur production en $\mathrm{CCP}^{10}$.

10. $50 \%$ en vente directe $(30 \%$ ferme, $15 \%$ domicile, $5 \%$ points de vente collectifs) et $50 \%$ en vente indirecte ( $45 \%$ bouchers, $5 \%$ GMS), constituant une moyenne de $20 \%$ du CA réalisé en CC. 


\begin{tabular}{|c|c|c|c|}
\hline & & Moyenne & $\begin{array}{c}\text { Nombre } \\
\text { de réponses }\end{array}$ \\
\hline \multirow{3}{*}{$\begin{array}{l}\text { Chiffre d'Affaires } \\
\text { (CA, en euros) }\end{array}$} & $\mathrm{CCL}$ & 124330 & 50 \\
\hline & RICA & 124617 & 132 \\
\hline & rapport CCL/RICA & 1 & \\
\hline \multirow{3}{*}{$\begin{array}{l}\text { Charges } \\
\text { (en euros) }\end{array}$} & $\mathrm{CCL}$ & 77590 & 49 \\
\hline & RICA & 84082 & 132 \\
\hline & rapport CCL/RICA & 0,92 & \\
\hline \multirow{3}{*}{$\begin{array}{l}\text { Excédent brut } \\
\text { d'exploitation } \\
\text { (= CA - charges) }\end{array}$} & $\mathrm{CCL}$ & 47787 & 49 \\
\hline & RICA & 40536 & 132 \\
\hline & rapport CCL/RICA & 1,18 & \\
\hline \multirow{3}{*}{$\mathrm{EBE} / \mathrm{CA}$} & $\mathrm{CCL}$ & 0,39 & 49 \\
\hline & RICA & 0,32 & 132 \\
\hline & rapport CCL/RICA & 1,24 & \\
\hline \multirow{3}{*}{$\begin{array}{l}\text { Annuités } \\
\text { (en euros) }\end{array}$} & $\mathrm{CCL}$ & 23402 & 43 \\
\hline & RICA & 21570 & 132 \\
\hline & rapport CCL/RICA & 1,08 & \\
\hline \multirow{3}{*}{ Annuités/EBE } & $\mathrm{CCL}$ & 0,65 & 42 \\
\hline & RICA & 0,58 & 132 \\
\hline & rapport CCL/RICA & 1,12 & \\
\hline \multirow{3}{*}{$\begin{array}{c}\text { Revenu disponible } \\
\text { estimé } \\
\text { (en euros) }\end{array}$} & $\mathrm{CCL}$ & 26562 & 42 \\
\hline & RICA & 18499 & 132 \\
\hline & rapport CCL/RICA & 1,44 & \\
\hline
\end{tabular}

\section{Définitions comptables retenues}

L'enquête $C C L$ réunit des données sur le chiffre d'affaires, les charges et les annuités bancaires ainsi que des données importées du RA sur la SAU, les UTA et les effectifs animaux. Pour ces trois der-nières données, les définitions sont univoques de sorte que la comparabilité est immédiate. Pour les trois premières informations, il est nécessaire de préciser le champ comptable retenu :

1.Les instructions aux enquêteurs $C C L$ précisent que le CA comprend les subventions à la différence de la notion de CA des données RICA. Nous avons donc redressé cette der-nière en y ajoutant les subventions.

2.Pour les charges, les enquêteurs CCL renseignaient le "montant total des charges suppor-tées par l'exploitation agricole", moins les dotations aux amortissements dans les cas d'exploitations ayant une comptabilité. Comme le poste annuités comprend les charges financières, elles ne sont pas intégrées dans cette variable. Pour les données RICA, ces charges correspondent donc à la formule suivante : charges d'exploitation + charges so-ciales de l'exploitant dotations aux amortissements

3.Pour les annuités bancaires, il s'agit dans l'enquête CCL comme dans les données RICA des annuités pour les emprunts long et moyen termes. II n'y a donc pas de problème de comparabilité.

Ces trois variables servent ensuite à calculer les soldes intermédiaires de gestion suivants (SIG) :

- Excédent brut d'exploitation $(\mathrm{EBE})=\mathrm{CA}$-charges (les charges sociales de l'exploitant sont comprises dans les charges)

- Revenu disponible estimé = CA-charges-annuités

Richard F., Chevallier M., Dellier J., Lagarde V., 2014 Conception et réalisation : Geolab UMR 6042 CNRS - Université de Limoges

Tableau 1 : Résultats d'exploitation et principaux soldes intermédiaires de gestion comparés des exploitations limousines en bovin viande (exploitations en CCP/ensemble des exploitations)

farms)

Comparison of operating results and main intermediate operating totals of beef farms in Limousin (short food supply chain farms/all

Concrètement, en Limousin, à chiffres d'affaires équivalents (autour de $124500 €$ en moyenne), elles dégagent un Excédent Brut d'Exploitation et un revenu respectivement supérieurs d'au moins $18 \%$ et $44 \%$ à ceux des exploitations commercialisant en filières longues. Le revenu par unité de travail annuel non salariée est également supérieur d'au moins $18 \%{ }^{11}$.

Les données disponibles permettent d'engager une rapide ébauche d'analyse comptable visant

11. Voir Chevallier, 2013b, pour une analyse plus détaillée. 
à détailler les composantes de ce différentiel. Globalement, les exploitations en CCP se caractérisent par une meilleure productivité : alors que les SAU et quantités vendues (estimées à partir de la PBS $^{12}$ moyenne) sont respectivement inférieures de 9 et $8 \%$ à celles des exploitations aux modes de commercialisation conventionnels, leur CA ramené au nombre de vaches nourrices est en revanche supérieur de $3 \%$. Il semble bien que la réintégration des fonctions de transformation et commercialisation au sein des exploitations en CCP permet de se réapproprier plus de valeur ajoutée. Parallèlement à ces recettes marchandes, les exploitations peuvent bénéficier de recettes non marchandes, sous formes de primes et subventions. Malheureusement, les bases de données disponibles ne permettent pas de les isoler au sein du résultat CA global des exploitations. Cependant, les exploitations en CCP et les autres bénéficient de primes différentes qui correspondent souvent à des modèles de productions euxmêmes distincts. Par exemple, en règle générale, les exploitations en bovin viande pratiquant les CCP « finissent leurs animaux » (c'est-à-dire qu'ils y sont élevés jusqu’à être abattus). A ce titre, elles peuvent bénéficier de primes supérieures à celles qui, en circuits conventionnels, privilégient l'élevage de broutards (« engraissés » par d'autres structures, parfois à l'étranger). Mais comme, inversement, ces exploitations spécialisées en broutard sont plus souvent localisées dans des zones à contraintes naturelles à l'origine de dispositifs spécifiques de soutien dans le cadre du $2^{\mathrm{e}}$ pilier de la PAC (Leyssenne, 2012), au final, l'impact de ces primes ne serait susceptible d'expliquer la bonne performance des exploitations en CCP que dans une proportion marginale.

En revanche, et de manière sensiblement moins attendue $^{13}$, le différentiel de résultat global pourrait s'expliquer en partie par les niveaux de dépenses des exploitations. En effet, les charges d'exploitation par

12. La PBS (production brute standard) est une estimation du chiffre d'affaires des exploitations réalisées à partir des données structurelles (surface agricole, cheptel, etc) en fonction de moyennes constatées. Elle sous-estime certainement la réalité du chiffre d'affaires des exploitations qui pratiquent les CCP puisque ces moyennes ne sont pas révisées en fonction des prix supérieurs que les exploitants peuvent dégager en vendant en CCP.

13. Les activités de transformation et commercialisation nécessitent en effet des dépenses supplémentaires. Toutefois, on peut faire l'hypothèse que les dépenses pour la production peuvent être moins élevées si les agriculteurs pratiquant les CCP s'inscrivent dans une agriculture plus «durable» vantant un mode de production plus « économe » car plus « autonome» (moins d'intrants). Nous remercions un relecteur pour cette remarque. vache nourrice en CCP sont inférieures d'au moins $6 \%$ à celles observées en filières longues, et ce, en intégrant des frais de personnel pourtant supérieurs (UTA supérieures de $22 \%$ en CC). A ce stade, les modalités de cette apparente meilleure maîtrise des charges courantes restent à établir mais plusieurs pistes mériteraient d'être suivies à l'occasion de recherches complémentaires. Dans quelle mesure les pratiques productives ou les investissements (les annuités sont sensiblement plus lourdes en CCP, cf. tableau 1) consentis par les producteurs en CCP bénéficient-ils à ces résultats? De même, il se pourrait que le profil des producteurs en CCP (beaucoup plus diplômés que la moyenne notamment, cf. infra) puisse influencer le résultat de leurs exploitations, a fortiori au sein d'un secteur peu institutionnalisé (Chevallier, 2013a), y compris en termes d'accompagnement des exploitants (Lagarde, 2004). De manière générale, la performance des exploitations en CCP repose donc peut-être davantage sur les caractéristiques individuelles des dirigeants et notamment leur profil entrepreneurial (Lagarde, 2006). Plus précisément, par définition dans le domaine des circuits-courts, la réussite du projet dépend de la capacité des producteurs à entrer en relation avec leurs clients afin, à la fois de réaliser des échanges économiques (trouver et approvisionner une clientèle) et d'optimiser ces échanges en fidélisant la clientèle et en garantissant une bonne marge.

En l'occurrence, ces éléments de réflexion relatifs aux liens entre performance économique des CCP et relations producteurs/clientèles ont fait écho à un certain nombre d'observations et résultats issus de diverses recherches conduites en Limousin depuis la fin des années 2000, notamment sur les nouvelles populations en milieu rural ${ }^{14}$. De cette confrontation entre premiers résultats et questionnements

14. La première, réalisée en 2010 , portait sur les relations entre environnement et populations néo-limousines à partir d'une étude de cas (Plateau de Millevaches) et a permis de constitué un corpus de 30 entretiens (personnes ressources) et 120 questionnaires auprès d'un échantillon d'anciens et nouveaux résidents (Richard et Dellier, 2011). Deux autres concernaient plus précisément les CCP et ont consisté en la production (en 2013), d'une part de 226 et 274 questionnaires passés auprès de consommateurs résidants, respectivement dans 4 communes du sud de la Creuse ([https://plateaudegentioux.wordpress.com/creation-dactivite-2/ relocalisation-de-lalimentation/], consulté le 22 janvier 2014) et au sein de l'agglomération de Limoges (document interne, licence de géographie de l'Université de Limoges). Enfin, les analyses quantitatives sont tirées d'une dernière étude constituée notamment d'un corpus de 1024 questionnaires (sur 5831 adressés par courrier) auprès de résidents des bassins de vie d'Argentat, Dun-Le-Palestel et Nexon (Dellier et Al. 2013). 
a émergé l'idée selon laquelle l'analyse strictement financière des facteurs de performance gagnerait à être enrichie d'une mise en contexte sociogéographique. En particulier, nous posons l'hypothèse selon laquelle les échanges économiques pourraient bénéficier d'une certaine proximité entre producteurs et consommateurs, de leur appartenance à un même groupe socio-culturel, voire de leur appartenance à une même classe sociale. Autrement exprimée, cette hypothèse renvoie à la notion et au processus de gentrification des espaces ruraux, lequel aurait un impact particulièrement significatif vis-àvis des CCP et de leur performance économique.

\section{GENTRIFICATION RURALE ET CIRCUITS COURTS DE PROXIMITÉ}

\section{Processus de gentrification rurale et transformations des campagnes}

Apparu dans la littérature scientifique sous la plume de R. Glass en 1964, le terme de gentrification décrivait alors les mutations sociales et urbanistiques à l'œuvre dans les quartiers centraux et péricentraux de la métropole londonienne des années 1950-60. Si la gentrification urbaine, à travers ses modalités et enjeux, a été à l'origine d'une abondante littérature dès les années 1980, la gentrification rurale a en revanche été plus lente à susciter l'intérêt des chercheurs en sciences sociales. En l'occurrence, les géographes et sociologues britanniques se sont progressivement emparés de cet objet dans les années 1980 (Parsons, 1980; Pacione, 1984 ; Little, 1987) et surtout 1990 (Phillips, 1993; Cloke et al., 1995; Smith, 1998). Puis les années 2000 ont été celles d'une diffusion sensiblement plus massive au sein de la sphère académique internationale (Curry et al., 2001 ; Ghose, 2004 ; Darling, 2005), y compris française (Raymond, 2005; Perrenoud, 2008, 2012; Cognard, 2010; Richard, 2010; Pistre, 2011, 2012).

Concrètement, la gentrification rurale pourrait être assez basiquement définie comme " processes whereby middle or service class households are moving into villages and displacing local, working class groups, and often in the process also refurbishing, extending and converting properties ${ }^{15}$ »(Phillips et

15. « processus par lequel les ménages des classes moyennes ou de service déménagent vers des villages, déplacent les populations locales appartenant aux classes populaires et, souvent dans la même dynamique rénovent, agrandissent ou convertissent les logements ». al., 2008). Dans le détail, considérant les singularités des espaces ruraux, sensiblement plus vides (parfois parce que vidés suite à une plus ou moins longue période d'exode), les travaux nuancent néanmoins l'importance du déplacement pour privilégier celle du remplacement des populations d'origine par de nouvelles catégories d'habitants, y compris par l'entremise des constructions neuves. De même, la multiplication des travaux empiriques a permis de conclure à une certaine hétérogénéité des populations susceptibles d'être qualifiées de gentrifieurs (Stockdale, 2010 ; Smith, Phillips, 2001 ; Richard, Dellier, 2011). Deux éléments, partiellement liés l'un à l'autre sont à souligner ici. En premier lieu, les gentrifieurs peuvent se caractériser en tant que tels au travers d'une série de capitaux (financiers, culturels, sociaux, etc.) et/ou de combinaisons variables de ces capitaux. De la même manière, selon les options de catégorisation socioprofessionnelle, les nomenclatures disponibles pour esquisser les contours des middle classes et/ ou des classes moyennes et supérieures, le regard porté au processus de gentrification variera (Hoggart, 1997; Phillips, 2007) En second lieu, la gentrification apparaît comme une notion relative en ce sens que les gentrifieurs peuvent être identifiés en tant que tels dès lors qu'ils disposent de capitaux supérieurs aux populations antérieurement implantées, faisant ainsi peser des pressions très inégales selon les profils respectifs des uns et des autres. De fait, il est exceptionnel qu'une publication mentionne explicitement le déplacement (effectif, physique) directement impliqué par l'implantation de gentrifieurs ruraux. Il s'agit plutôt d'une mise et d'un maintien à l'écart des populations les plus modestes. Dès lors, considérant ces divers éléments, les auteurs sus cités évoquent des types originaux de gentrifieurs, tels que les marginal gentrifiers, les super gentrifiers, main stream, etc. Par ailleurs, des approches plus théoriques ont enrichi cette définition en précisant par exemple la nature (et son évolution) des espaces de la gentrification, en particulier au regard des étapes ou de l'état d'avancement du processus de gentrification. Bryson et Wickock (2010) ou Hines $(2010,2012)$ ont ainsi pu souligner le fait que les flux migratoires, vecteurs du changement social, visaient les «predominantly working class, primary production-oriented rural communities, economies 
and labour markets ${ }^{16} »$ (Argent et al., 2009). De fait, en même temps qu'ils font l'objet de cette gentrification, les territoires concernés voient leurs fonctions évoluer pour progressivement favoriser leurs dimensions récréatives : "gentrification is not only a displacement of social classes and persons, but also brings changes in leisure and retail activities, consumption patterns $^{17}{ }^{17}($ Solana-Solana, 2010). Plus précisément, les chercheurs font le constat selon lequel il $\mathrm{y}$ a « a tendency to displace longtime residents, commodify space, and involve a shift in landscapes of production to landscapes of consumption ${ }^{18}$ " (Bryson et Wyckoff, 2010 : 55). Cet argument selon lequel les espaces ruraux seraient devenus ou deviendraient à la fois des espaces et/ou des biens de consommation est semble-t-il unanimement partagé par les chercheurs, et ce, par-delà les nuances, voire exceptions possibles, liées à de quelconques spécificités géographiques.

Précisons par ailleurs, que la nature (ou « l'expérience de la nature » pour certains, cf. Hines, 2012), les paysages et, plus globalement « l'environnement » au sens large, jouent un rôle fondamental dans cet ensemble de transformations (Richard, 2010; Richard, Dellier, 2011), au point d'inciter D. Smith et D. A. Phillips à évoquer la notion de greentrification en lieu et place de celle de gentrification rurale (Smith, Phillips, 2001), soulignant ainsi les interactions entre stratégies résidentielles des gentrifieurs et la nature (ou sa représentation), aussi bien en amont qu'en aval de leur installation proprement dite. Plusieurs recherches ont porté sur les conséquences de cette greentrification vis-à-vis des activités susceptibles d'avoir un impact environnemental, y compris paysager, dont naturellement l'agriculture. En l'espèce, au regard de leur contribution supposée en termes de bonnes pratiques environnementales et de développement durable, les CCP pourraient mériter une attention toute particulière.

\footnotetext{
16. « des communautés, des économies et des marchés du travail ruraux majoritairement populaires et plutôt orientés vers le secteur primaire. » 17. « la gentrification n'est pas seulement un déplacement de personnes et des classes sociales, elle apporte également des mutations dans les loisirs, les activités commerciales et les modèles de consommation. »

18. " une tendance à déplacer les anciens résidents, à "marchandiser" l'espace et impliquer une évolution des paysages de production vers des paysages de consommation ».
}

\section{Gentrification rurale et circuits courts de proximité agroalimentaires en Limousin}

Associer le processus de gentrification rurale et les CCP agroalimentaires ne va pas spontanément de soi, notamment parce que les CCP sont très souvent présentés et analysés dans une perspective très urbano-centrée ${ }^{19}$. Une étape préalable à la suite de l'argumentation sur les classes sociales consisterait donc ici à nuancer, en tout cas dans le contexte limousin, cette articulation de type ville/campagne qui structurerait nécessairement les CCP.

\section{Un regard moins urbano-centré sur les CCP}

De fait, le Limousin présente un certain nombre de caractéristiques géographiques qui ne sont peut-être pas étrangères à nos interrogations et positionnement. Outre le fait d'être peu peuplée (742 800 habitants en 2010), la région est fortement polarisée, impliquant un profond déséquilibre démographique. En résumé, plus des deux-tiers de la population sont concentrés sur moins du tiers sudouest du territoire, autour d'un axe Limoges-Brivela-Gaillarde. Inversement, à l'exception de quelques petites unités urbaines, le reste du Limousin est très peu occupé, en particulier la montagne limousine, mais pas seulement. Les pôles urbains de Limoges et Brive abritent $35 \%$ de la population totale. En dehors de ces deux pôles, le Limousin ne compte que 4 villes de plus de plus de 10000 habitants, 7 si l'on prend le seuil des 5000 habitants. En outre, il n'est pas inutile d'ajouter que les périphéries immédiates des deux principales agglomérations offrent elles aussi un visage indiscutablement marqué par le sceau de la "ruralité », avec par exemple des densités inférieures à 20 et même 10 habitants $/ \mathrm{km}^{2}$ pour des communes pourtant éloignées de moins de quinze minutes des villes-centres. Autrement exprimé, une grande partie de la clientèle potentielle des producteurs recourant aux CCP réside en campagne, majoritairement « à proximité » des producteurs dont la distribution géographique est quant à elle relativement homogène sur l'ensemble du territoire régional (figure 2). En ce sens, l'enquête CCL

19. Cf. par exemple le dossier spécial de Norois (224, 2012) consacré aux Circuits courts et aux agricultures paysannes. Les contributions respectives de J. Blanc et M. Houdard et al. s'appuient ainsi sur des cas d'étude périurbains et/ou plus généralement à l'interface ville/campagne (Blanc, 2012; Houdard et al., 2012). 


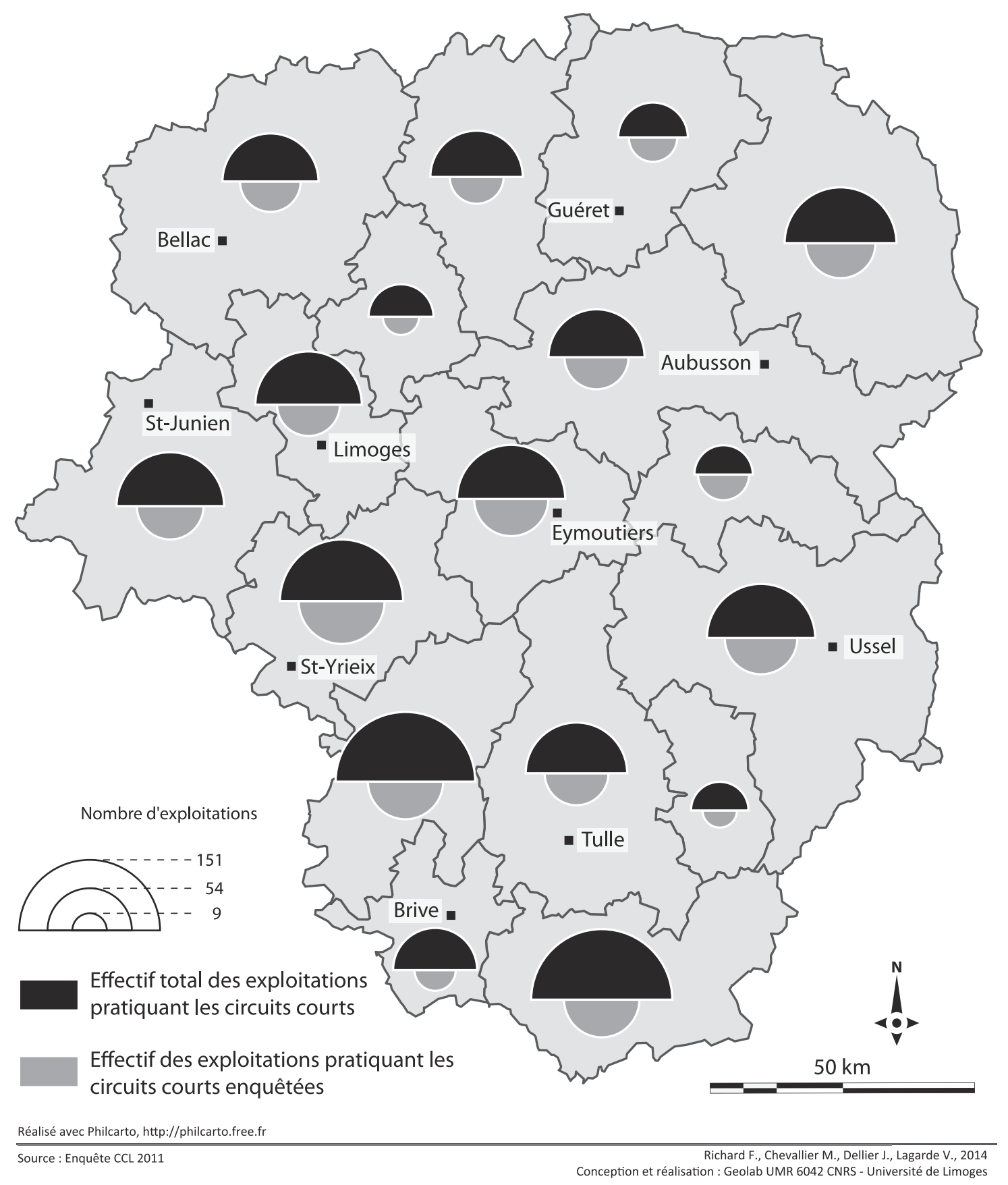

Figure 2 : Les exploitations pratiquant les circuits courts par «pays» dans le Limousin. Effectifs comparés entre l'enquête CCL et le RICA Number of farms involved in short food supply chain by Limousin "pays". Comparison between the results of the additional survey and of the farm accountancy data network

apporte quelques autres éléments nous invitant à nuancer la dimension ville-campagne des CCP en Limousin. Ainsi, seulement $38 \%$ des producteurs y recourant écoulent tout ou partie de leur production sur les marchés de plein vent dont, sans compter les marchés de producteurs au caractère beaucoup plus saisonnier, moins de la moitié se tiennent dans une ville ou un gros bourg ${ }^{20}$. Inversement, $70 \%$ des producteurs pratiquent la vente à la ferme et $33 \%$ via les commerçants détaillants dont la distri-

20. La localisation des marchés de plein vent a pu être identifiée sur la base des déclarations en préfectures de département (en 2012). 
bution géographique est loin d'être exclusivement urbaine. Autre illustration, le nombre de kilomètres parcourus par les agriculteurs en CC n'est pas corrélé à la distance séparant leurs exploitations des concentrations urbaines ${ }^{21}$. Dernier exemple, sur la trentaine d'AMAP et autres formes de partenariats producteurs/consommateurs recensées en 2012 en Limousin $^{22}$, neuf se trouvaient à Limoges, Brive ou Tulle, quatre dans des communes urbaines de très moindre rang (La Souterraine, Uzerche, Ussel, Bellac) : les deux-tiers de ces structures étaient donc localisées dans des zones indiscutablement rurales.

\section{De nouveaux habitants, de nouveaux «consom'acteurs »}

Pour revenir plus précisément à la gentrification rurale et aux liens possibles à établir avec les CCP, il faudrait distinguer les facettes respectivement démographique et socioculturelle du phénomène. Concernant la première, l'arrivée de nouveaux habitants constitue d'abord une augmentation (à défaut un maintien dans les territoires à l'évolution démographique encore incertaine voire déclinante) de la demande locale. Dans le périurbain et/ou rural proche de Limoges, les nouvelles populations sont composées en majorité de ménages originaires du reste du Limousin, et singulièrement des deux centres d'agglomération. En dépit de la présence de ménages relativement modestes (qui restent néanmoins en capacité d'accéder à la propriété), ces zones périphériques de Limoges et Brive se caractérisent par les revenus moyens les plus élevés de la région. Inversement, en dehors de ces deux espaces, c'est-à-dire dans environ $80 \%$ des communes de la région, pour les dernières enquêtes de recensement, les néo-Limousins ont représenté plus de la moitié des nouveaux habitants. Compte tenu du tableau démographique dressé plus haut, le poids relatif de ces nouveaux résidents au sein des campagnes limousines y est d'autant plus fondamental : il n'est ainsi pas rare que les néo-Limousins implantés au cours d'une période intercensitaire (1999-2006 par

21. En outre la distance parcourue pour la vente la plus courante est en moyenne de 35 kilomètres, en dehors des exploitants qui ne pratiquent que la vente à la ferme et n'ont donc aucun kilomètre pour la vente, la moyenne passant à $26 \mathrm{~km}$ quand on les réintègre (Source : enquête CCL).

22. D'après une liste de la fédération régionale des agriculteurs biologiques du Limousin, Gablim, établie dans le cadre d'un groupe de travail sur les CCP. exemple) pèsent plus de 15 ou $20 \%$ de la population communale, et parfois plus encore (Dellier $e t$ al., 2013) Quoi qu’il en soit, qu'il s'agisse des néoruraux des marges limougeaudes et brivistes ou des néo résidents issus de migrations interrégionales, considérés en tant que consommateurs ils présentent l'avantage de disposer en général de revenus sensiblement supérieurs à ceux des populations locales et/ou antérieures ${ }^{23}$.

Concernant ensuite le profil socioculturel des migrants et potentiels gentrifieurs ruraux, il apparaît là encore relativement distinctif. Si une analyse des flux migratoires en CSP des actifs confirme une nette différence entre populations locales et nouveaux résidents, le décalage est plus marqué encore à travers le niveau de qualification et de diplôme, permettant ainsi d'intégrer les ménages retraités (figure 3). Par ailleurs, les trajectoires migratoire et résidentielle de ces néo-Limousins sont le fruit d'un certain nombre de motivations qui diffèrent des préoccupations des populations locales et sont cohérentes avec une demande de CCP. Les différentes enquêtes réalisées ces dernières années auprès de nouveaux résidents ont ainsi mis en évidence, pour les trois principales, des motivations d'ordre environnemental et/ou social (liées au cadre de vie rural et de type «villageois »), plutôt que d'ordre économique et professionnel. À cet égard, la littérature regorge d'illustrations de projets migratoires fondés sur des représentations, voire des formes d'idéalisation des campagnes et de la vie en campagne. Outre le fait d'être parfois en décalage avec la réalité, ces représentations sont en revanche plus souvent encore en décalage avec celles que les populations locales portent sur le même territoire. Pour les terrains limousins investigués, les préoccupations environnementales des néo-Limousins étaient ainsi plus affirmées que celles des populations locales, répondant en cela assez fidèlement à la distinction opérée dans d'autres contextes ruraux entre des visions qualifiées par exemple de « romantique » et "moderniste » du présent et de l'avenir des campagnes (Marsden et al., 1993). Entre autres thématiques, cette opposition est apparue à propos du recours aux CCP.

23. Au point d'avoir contribué à un rattrapage partiel entre revenus moyens des ménages ruraux et urbains à l'échelle régionale au cours des années 2000 (calcul des auteurs, source : DGI). 


\section{La population ayant un diplôme supérieur à bac +2 en 2006 en Limousin}

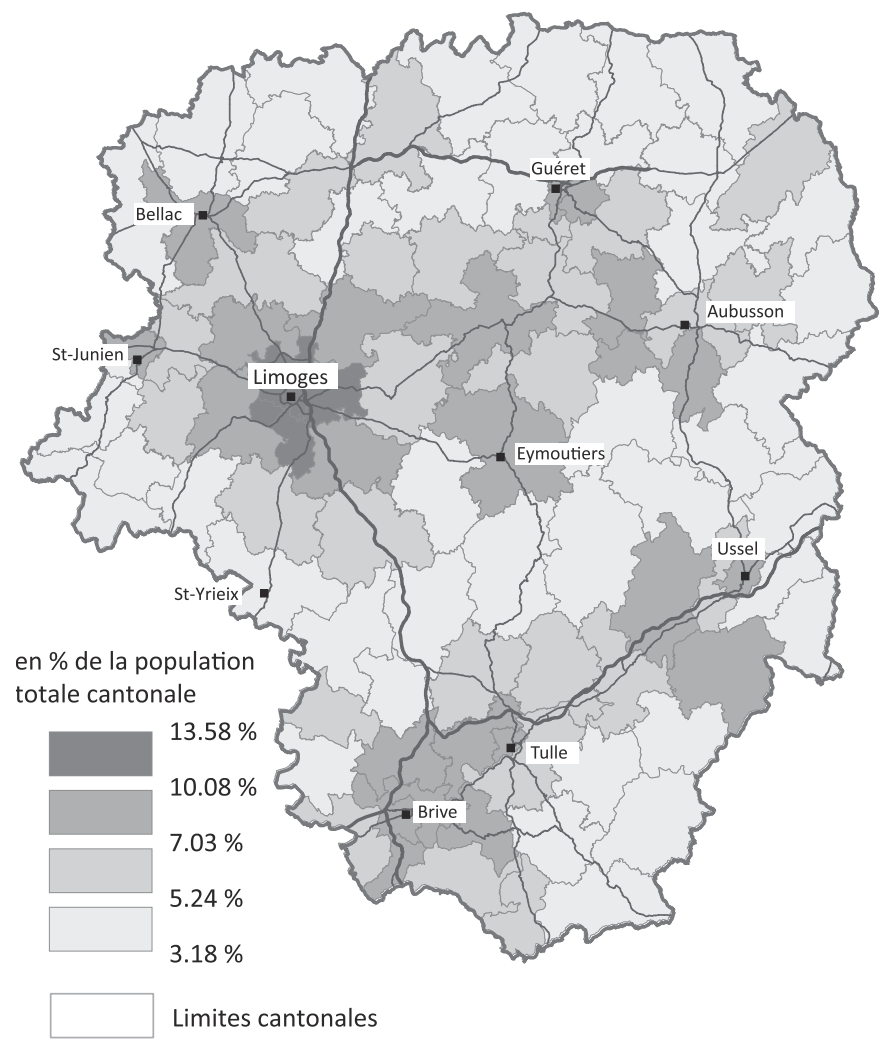

Les néo-limousins implantés entre 1999 et 2006 et au moins diplômés d'un bac +2

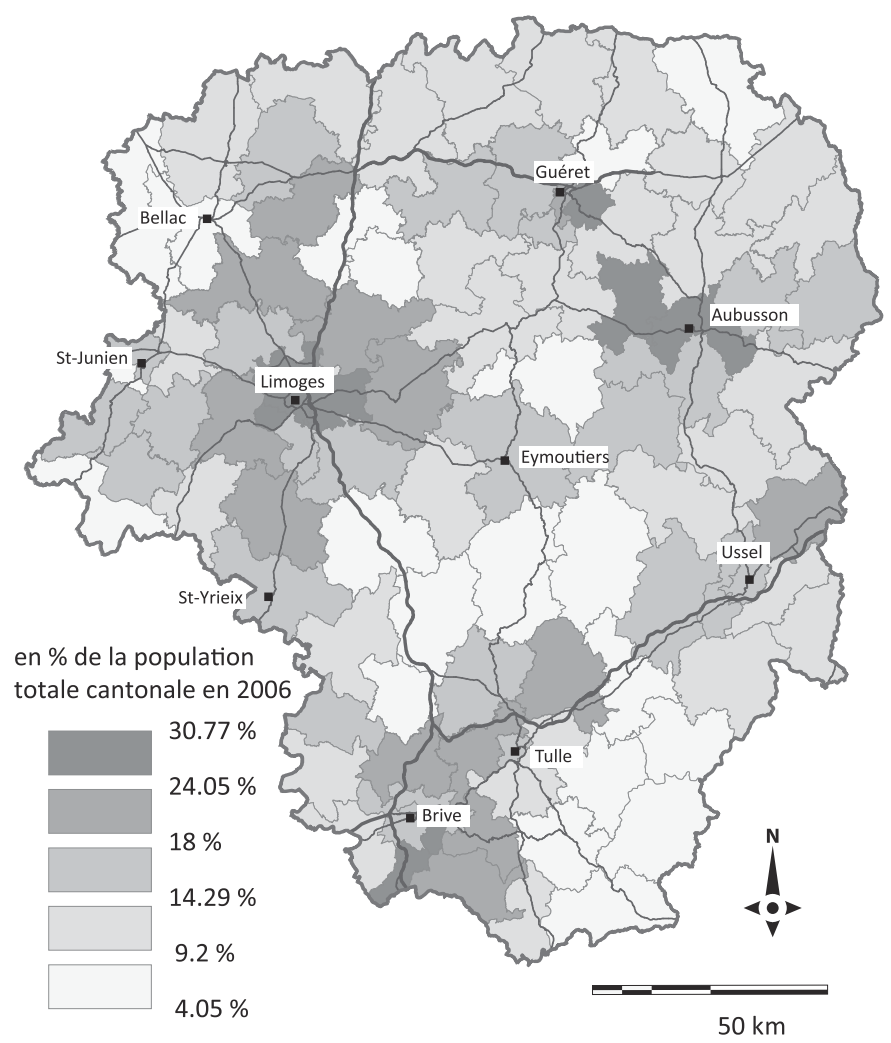

mode de discrétisation utilisé : méthode de jenks

Réalisé avec Philcarto, http://philcarto.free.fr

Source : INSEE, Base de données formation - diplôme 2006, MIGCOM 2006

Richard F., Chevallier M., Dellier J., Lagarde V., 2014 Conception et réalisation : Geolab UMR 6042 CNRS - Université de Limoges

Figure 3 : néo-Limousins et recompositions socioculturelles des espaces ruraux : l'exemple des diplômés (diplômes supérieurs à bac+2) Newcomers in Limousin and sociocultural restructuration of rural areas: the example of graduates (Baccalaureat +2 and more)

En l'espèce, les premiers résultats obtenus en Limousin sont conformes à ceux qui ont pu être produits par ailleurs et qui attestent d'une relation aux CCP socialement différenciée, que ce soit dans les AMAP (Allen et al., 2003, 2006 ${ }^{24}$; Mundler, 2007; Dufeu et Ferrandi, 2012; Paranthoën, 2013) ou plus largement parmi les consommateurs potentiellement intéressés par les CCP (Fournier et Sirieix, 2009). Concrètement, les différentes enquêtes confirment le fait que les ménages disposant des revenus les plus élevés et/ou appartenant aux catégories socioprofessionnelles supérieures (Cadres, Professions Intellectuelles Supérieures et dans une moindre mesure les Professions Intermédiaires) ont un recours sensiblement plus fréquent que les

24. « Les membres des CSA sont majoritairement de milieu social élevé, ont de hauts niveaux d'éducation et sont "caucasiens" »(Allen, op. cit.). catégories plus populaires aux productions agricoles locales. Les questionnaires passés au sein du PNR Millevaches permettent de compléter l'analyse au regard d'autres critères. Par exemple, plus de $60 \%$ des néo-Limousins interrogés déclarent recourir le plus possible aux produits agricoles locaux contre $43 \%$ des populations autochtones. Analysée par le prisme du niveau de diplôme, plus pertinent pour raisonner en termes de capital culturel, la distinction est plus forte encore : les deux-tiers des « bac +3 et plus » disent recourir le plus possible aux CCP, contre 33,3 \% des "sans diplômes ». De manière plus générale, les revues de littérature, qu'elles soient françaises (Chiffoleau, 2008) ou anglo-saxonnes (Deverre et Lamine, 2010), soulignent le fait que les systèmes agro-alimentaires alternatifs (S3A) font l'objet d'un nombre d'ar- 
ticles scientifiques disproportionné par rapport à leur importance quantitative réelle dans le paysage agroalimentaire. Deverre et Lamine (2010) expliquent ce phénomène par l'intérêt personnel des chercheurs pour ces formes de consommation, au point que "nombre d'auteurs sont impliqués dans des S3A, les accompagnent ou en sont proches » (ce qui est d'ailleurs le cas des auteurs de la présente contribution), confortant ainsi l'idée d'une consommation de classe de diplômés du supérieur.

Au-delà, d'une part, de leurs représentations singulières (et d'ailleurs pas forcément ni unanimes ni homogènes) de l'environnement ou de l'espace rural, et d'autre part de leur capital culturel, les gentrifieurs ruraux se caractérisent également par leur capacité à projeter sur leur territoire les représentations qu'ils en ont. Les recherches relatives à la gentrification rurale ont montré qu'en mobilisant leur capital culturel, leur capital économique, leur capital social, en investissant les champs associatifs et politiques locaux, en finissant parfois par représenter la majorité de la population, les gentrifieurs parviennent progressivement à modeler leur campagne à l'image de la représentation qu'ils s'en étaient forgée, y compris en Limousin. En l'espèce, rapportées aux CCP, les AMAP et autres formes innovantes de partenariats entre producteurs et consommateurs constituent de parfaites illustrations de cette aptitude à concrétiser leurs désirs de campagne à la fois authentique et durable. Au même titre que Paranthöen (2013) a pu le décrire pour son cas d'étude en Pays de la Loire, les observations conduites en Limousin viennent attester que ces formes de CCP, certes très minoritaires, sont le plus souvent à l'initiative de, animées par, et de fait, au service de groupes socioculturels bien spécifiques qui, sans que ce soit un objectif en soi, s'en saisissent comme d'une pratique socialement distinctive (Bourdieu, 1979). Par ailleurs, la construction de ces partenariats est d'autant plus facilitée que le profil des agriculteurs concernés n'est pas toujours très éloigné de celui (de tout ou partie) de leurs clients.

\section{Producteurs et "gentriculteurs"}

Les recherches consacrées à la gentrification rurale opposent régulièrement les gentrifieurs aux populations locales, et notamment aux agriculteurs en ce que les représentations, objectifs et pratiques des uns et des autres ne sont pas toujours parfaitement compatibles, loin s'en faut (Argent et al. 2009; Hines, 2010). Pour sa part, Sutherland propose une lecture originale de la gentrification rurale pour s'interroger sur le retour du gentleman farmer, et tenter de conceptualiser ce que pourrait être la gentrification de l'agriculture au Royaume-Uni, en tout cas de certaines catégories d'agriculteurs, pratiquant notamment le hobby farming (Sutherland, 2012). Sans forcément suivre très exactement les pistes ouvertes par Sutherland, la place et le rôle des producteurs dans le processus de gentrification rurale et les liens éventuels entre celle-ci et les CCP agro-alimentaires méritent d'être interrogés.

Pour commencer, il est sans doute utile de revenir sur les flux migratoires à l'origine de la gentrification - encore très partielle - des campagnes limousines. Chaque année, ils sont en partie alimentés par des agriculteurs, néo- et futurs agriculteurs qui choisissent le Limousin pour y reprendre ou y créer une exploitation (environ une centaine par an entre 2003 et 2008 par exemple ${ }^{25}$ ). A cet égard, l'expérience de Rémy ${ }^{26}$, ce nouveau résident interviewé à l'occasion d'un terrain en sud Creuse, est relativement banale:

" On est arrivé à la naissance de mon fils ainé, on vivait déjà à la campagne, mais ici c'était tellement peu habité que c'était attirant, vierge d'industrie, vierge de pollution [...]. Je voulais changer de vie, arrêter de bosser comme éducateur et je me suis mis à faire du marâ̂chage en bio et plusieurs autres activités » (néo-résident actif, Vassivière plateau).

À l'image de Rémy, les néo-agriculteurs et les néo-Limousins sont précisément deux profils surreprésentés parmi les producteurs limousins recourant aux $\mathrm{CCP}^{27}$. Ils le sont au même titre que les femmes, les jeunes, les individus diplômés et enfin ceux qui ont pu occuper des emplois qualifiés sans lien aucun avec l'agriculture avant leur installation (Chevallier, 2013a; Chevallier et al., à paraître). Autrement dit, ces caractéristiques sont relative-

25. Calculs des auteurs, source INSEE, fichiers MIGCOM.

26. Les citations sont anonymées.

27. Alors que $24 \%$ des exploitants en circuit court ne sont pas issus du monde agricole (contre $16 \%$ des exploitants n'usant pas des CC, Chevallier, 2013a), ce taux monte à respectivement $41 \%$ et $32 \%$ pour ceux qui se sont spécialisés dans le commerce sous la forme de paniers ou en point de vente collectif (source : Enquête CCP). 
ment semblables à celles des gentrifieurs tels qu'ils sont habituellement décrits dans la littérature et qu'ils peuvent être identifiés en Limousin. Pour sa part, si Paranthöen ne parle pas explicitement de gentrification de la profession, ses recherches déjà mentionnées sont susceptibles de nous éclairer quant aux modalités et conséquences de ce qu'il qualifie plutôt d'embourgeoisement. Ce dernier résulterait certes du renouvellement d'une partie de la population agricole par l'entremise des néo-agriculteurs décrits ci-dessus, mais il serait également le fruit de l'allongement et de la diversification des parcours de formation des agriculteurs issus de la profession. Cette double évolution de la profession entraînerait l'émergence d'une catégorie singulière de producteurs, plus fortement dotés en termes de capital culturel, là encore relativement comparable à celui des nouveaux résidents qualifiés de gentrifieurs.

\section{Rencontres et alliance de gentrifieurs et producteurs en $\mathrm{CCP}$}

Si nous ne sommes pas en mesure de défendre ici l'idée selon laquelle ils constitueraient un seul et même groupe social, voire une même classe sociale, pour reprendre un terme cher aux spécialistes de la gentrification (notamment rurale), à l'image de ce qu'a pu observer Ripoll vis-à-vis des consommateurs et producteurs d'AMAP (Ripoll, 2010), les profils socioculturels des gentrifieurs et des producteurs semblent néanmoins converger. Dans cette perspective, ils tendraient à adhérer à certaines valeurs communes (ou plus globalement à des systèmes de valeurs) et ainsi à partager diverses pratiques socialement distinctives, dont le recours aux CCP. À l'image de ce que Perrenoud (2012) a pu montrer à l'issue de ses recherches relatives aux artisans du bâtiment et agents ${ }^{28}$ de la gentrification dans les Corbières, ces valeurs communes auraient pour première conséquence de faciliter les rencontres et la compréhension entre producteurs et gentrifieurs. Elles permettraient par ailleurs de sceller une forme d'alliance entre eux, alliance fondée sur des intérêts partagés, en tous cas très complémentaires.

La commercialisation en CCP, puisqu'elle se déroule à la marge des principaux lieux du com-

28. Pour reprendre une expression usuelle dans le champ, notamment à la suite de l'article de Phillips (1993: 134). merce alimentaire, nécessite que les producteurs et les consommateurs s'appuient sur leurs propres réseaux. L'appartenance à un même groupe social est donc un facteur de réussite des circuits courts pour deux raisons. D'une part, elle remplit une fonction de mise en relation : l'émergence et la mise en commun de réseaux sociaux sur lesquels des partenariats producteurs/consommateurs peuvent se construire et se pérenniser. D'autre part, elle favorise la compréhension en ce sens que l'appartenance à une classe sociale exprime et induit également le fait d'être sensible à certains codes, à certains messages véhiculant des goûts, des appétences, des valeurs à travers lesquels s'exprime consciemment ou non un sentiment d'appartenance (Bourdieu, 1979). Très concrètement, en ce qui concerne les CCP, les observations de terrains confirment l'existence, pour ne pas dire l'omniprésence de ces signaux qui, considérés individuellement peuvent paraître anecdotiques, mais qui sont de fait très signifiants. Pour être très prosaïque, citons l'exemple des tarifs quelquefois pratiqués (en particulier sur les produits transformés) et des modes soit de commercialisation (en colis de 5 à $10 \mathrm{~kg}$ pour la viande notamment) soit de paiement, parfois adoptés dans le cadre de partenariats producteurs/consommateurs ${ }^{29}$, qui sont d'emblée socialement sélectifs. Il peut aussi bien s'agir du nom d'une AMAP à consonance latine, d'une boutique de producteurs à la décoration plus proche de celle de l'épicerie fine que de l'étal traditionnel en marché de plein vent, au panier « logoté et markété », en toile de jute ou coton bio (figure 4). De fait, quels qu'ils soient, ces marqueurs semblent être très efficaces : "sur le marché, ils ne sont pas forcément bio, mais bon déjà quand on voit comment est le stand et comment sont les producteurs, on ne se trompe pas trop » (extrait d'entretien avec Jean-Yves, consommateur en CCP).

Pour caricaturaux qu'ils puissent paraître, ces exemples d'expressions non verbales (mais qui peuvent l'être à l'occasion) d'identité socioculturelle et de reconnaissance sont évidemment (co-) produits par les producteurs. Pour une partie d'entre eux, les entretiens ont montré qu'il s'agissait d'adapter précisément leurs pratiques commerciales à la «clientèle » qu'ils visent. Mais pour d'autres, la réponse à cette demande spécifique apparaît d'autant plus

29. Il arrive que les paiements soient semestrialisés ou trimestrialisés, rendant de fait socialement sélective l'adhésion à la démarche collective. 


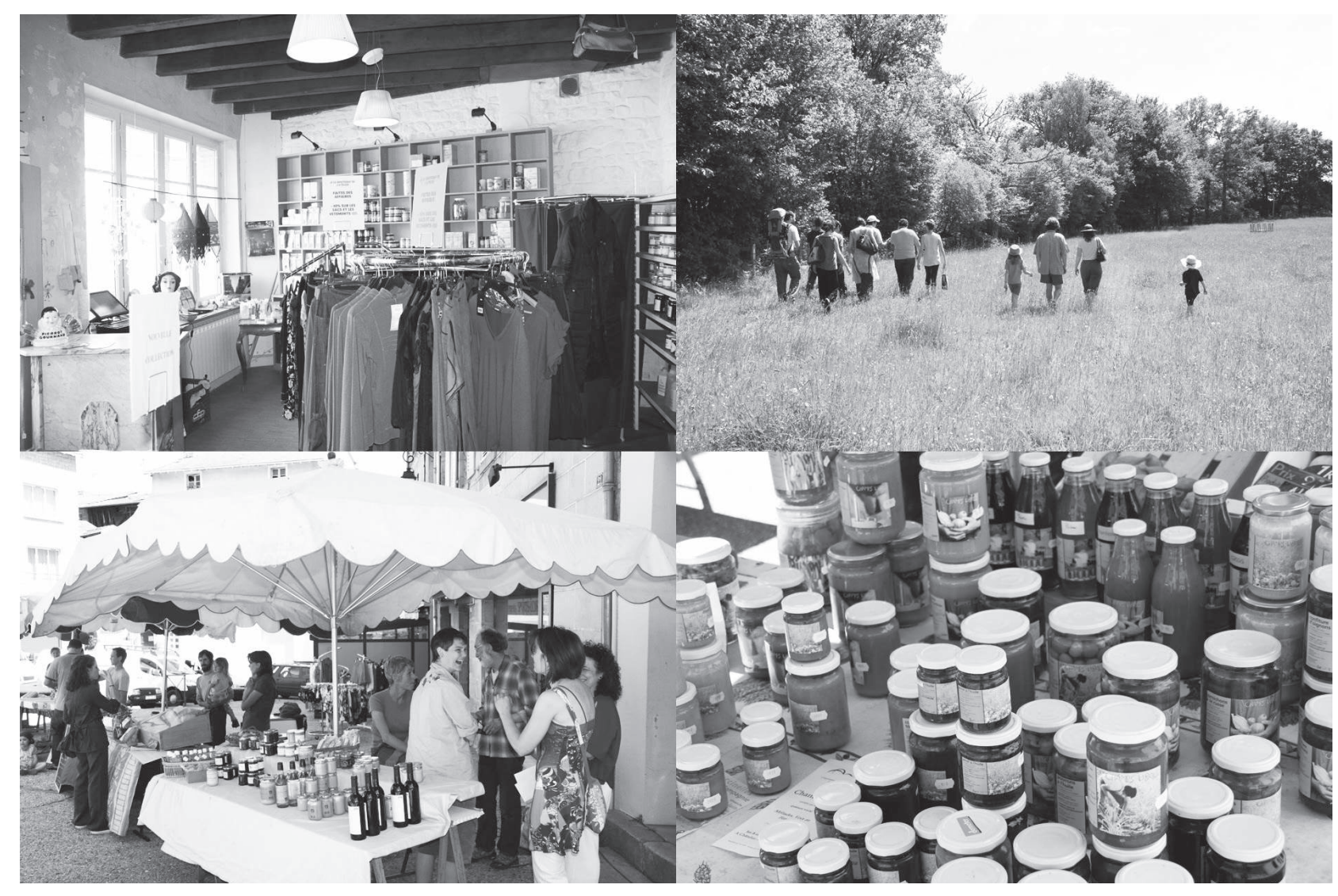

Figure 4 : Codes, marqueurs et proximité socioculturelle entre producteurs et consommateurs (exemples de marketing et de mise en scène de produits transformés, sociabilisation producteurs consommateurs sur l'exploitation et sur le marché)

Codes, signs and sociocultural proximity between farmers and customers (examples of marketing and valorisation of transformed products, socialization between producers and customers on the farm and in street market)

naturelle et facile à satisfaire qu'elle est émise par des consommateurs issus d'un groupe social auquel ils appartiennent eux-mêmes ou dont ils peuvent se sentir proches. La relation de cause à effet peut être discutée, mais cette production d'une « esthétique des CCP » pourrait même éclairer le fait que les charges d'exploitation soient plus lourdes pour les agriculteurs les plus diplômés (données CCL, population des exploitations en bovin viande) : les producteurs semblent prêts à engager des dépenses visant à soigner la présentation de leurs produits.

Par ailleurs, au-delà de ces indices et marqueurs d'appartenance et de reconnaissance mutuelle, les diverses enquêtes produites en Limousin montrent que producteurs et consommateurs sont motivés par des valeurs fondamentales, que les uns et les autres parviennent à concilier de manière assez pragmatique. Ainsi, les ruraux consommateurs de productions agricoles locales interrogés en Limousin se disent en recherche de qualités gus- tatives et sanitaires. De leur côté, parmi les motivations avancées par les producteurs figure la volonté de changer de mode de production et d'obtenir une meilleure reconnaissance de la qualité du travail fourni, autant du point de vue du produit que des modes de production (la qualité ayant un coût ${ }^{30}$, elle pourrait là encore peser sur les charges). De la même manière, les uns cherchent à renouer le contact avec les producteurs pour "savoir d'où vient ce qu'on mange » et prennent plaisir à se rendre à la ferme $(70 \%$ des personnes interrogées dans les enquêtes). Les autres, forts de cette relation directe, s'assurent une certaine autonomie et liberté d'action vis-à-vis des institutions sectorielles ou commerciales (cf. Chevallier et al. : à paraître). Enfin, si les premiers peuvent être animés par

30. On retrouve ainsi un résultat classique de l'économie industrielle, à l'origine de l'économie des proximités (RERU, 1993), qui distingue le positionnement économique sur les coûts de celui qui privilégie la qualité (Colletis et Pecqueur, 2005). 
une démarche citoyenne et même quasi militante dans certains cas $^{31}$, démarche visant à favoriser le développement (durable) de leurs territoires, pour les seconds, les CCP sont présentés comme un moyen de maintenir l'emploi local (y compris le leur), etc. Pour concilier cet objectif commun, les producteurs peuvent ainsi compter sur le capital économique des gentrifieurs, capital que ces derniers consentent d'autant plus aisément à investir qu'il concourt à fabriquer ou conforter la campagne qu'ils ont justement choisi d'investir.

\section{Conclusion}

L'enquête Circuits courts en Limousin et son recoupement avec certaines données comptables ont permis de réaliser une comparaison relativement inédite des performances et revenus entre exploitations pratiquant ou non les CCP à une échelle régionale. Les exploitants spécialisés en bovin viande atteignent en moyenne des revenus par personne plus élevés lorsqu'ils pratiquent les CCP que lorsqu'ils ne les pratiquent pas. Il s'agit en cela d'un apport majeur comparé aux résultats disponibles dans la littérature, du double point de vue de sa dimension comparative (entre CCP et non CCP) et de la variété des données comptables exploitées. Malheureusement, et en dépit de la lourdeur du dispositif mis en œuvre pour constituer le corpus, en particulier pour produire l'enquête CCL, il n'est statistiquement pas possible de l'exploiter plus avant. Nous empêchant ainsi, au-delà des quelques pistes évoquées (par exemple sur le différentiel de productivité ou la maîtrise des charges), de mieux identifier et déconstruire les facteurs explicatifs de cette performance économique supérieure. Dans cette perspective, la question de la coordination entre les acteurs étant centrale aux dynamiques des CCP, nous nous sommes interrogés sur les conditions, modalités et effets des rencontres entre producteurs et consommateurs. Le constat selon lequel d'une part, les consommateurs en CCP sont surreprésentés parmi les Limousins les plus quali-

31. Ainsi, sur l'un des terrains, à la question des motivations, les personnes interrogées ont répondu à $45 \%$ (ou $64 \%$ si on prend en compte les réponses « oui complètement » et « oui mais surtout pour la qualité des produits et permettre aux producteurs vivre sur territoire ») se considérer comme " consom'acteurs ». Dans les détails, cette réponse a été formulée par $51 \%$ (ou $69 \%)$ des diplômés d'un bac +3 et + contre à peine plus de $30 \%$ (ou $50 \%$ ) des non titulaires du bac et sans diplôme. fiés et les actifs appartenant aux catégories socioprofessionnelles supérieures, et d'autre part que les producteurs en CCP sont également plus diplômés que la moyenne, nous a incités à réfléchir à leur appartenance possible à un même " groupe » socioculturel au regard de capitaux et critères culturels, symboliques, économiques et sociaux : les performances économiques supérieures à la moyenne des exploitants pratiquant les CCP peuvent provenir d'une capacité des producteurs à entrer en contact avec une clientèle disposée à s'approvisionner en CCP. Par ailleurs, en Limousin, la dynamique des CCP ne semble pas aussi polarisée par les centralités urbaines que dans d'autres régions. Au contraire, les CCP limousins sont fortement marqués par leur caractère rural. De ces divers constats est née l'hypothèse d'un lien possible entre changement social des campagnes limousines et CCP. Plus précisément, les éléments théoriques et empiriques mobilisés ont montré que le processus de gentrification rurale était susceptible d'expliquer au moins pour partie la bonne performance économique des exploitations recourant aux CCP. De par son caractère inductif, la recherche présentée ici peut être considérée comme ayant surtout été l'opportunité de poser quelques jalons de travaux futurs. En effet, certaines faiblesses et lacunes devront être comblées : tous les secteurs productifs sont-ils concernés par les bons niveaux de performance économique des exploitations en CCP? L'idée d'une organisation plutôt ruralo-centrée des CCP est-elle si spécifique au Limousin? Le lien entre gentrification rurale et performance des CCP concerne-t-il tous les types de CCP ? Etc. D'une certaine manière, ces interrogations (non exhaustives) confortent notre conviction selon laquelle l'articulation conceptuelle et empirique entre circuits-courts et gentrification rurale constitue une piste scientifiquement prometteuse. De fait, le lien entre dynamique des circuits courts et profils sociaux des territoires n'est pas forcément si atypique dans la littérature. S'appuyant ainsi sur le cas de programmes américains d'approvisionnement des écoles par des fermiers locaux (Farm-to-school programs) qui s'avèrent surtout développés par des collectivités de milieux aisés, Allen et Guthman (2006) concluent par exemple que «l'apologie de la relocalisation peut aussi menacer les principes d'équité sociale ». D'autres auteurs (Winter, 2003; Watts et al., 2005; Feagan, 2007) estiment même que ces 
organisations peuvent devenir un prolongement du système libéral ${ }^{32}$ quand elle aboutissent à la mise en concurrence des communautés entre elles, créant ainsi paradoxalement de nouvelles formes d'inégalités entre celles qui réussiraient à assumer ce transfert de prérogatives de l'Etat (désengagement), pour des raisons sociales, économiques, géographiques ou politiques, et celles qui n'y parviendraient pas. On serait alors loin des contributions annoncées des CCP à la réduction des inégalités sociales - la " démocratie alimentaire » selon Lang (2000) grâce à une forme de résistance citoyenne censée procurer aux ruraux les moyens de réagir aux conditions économiques et sociales qu'exercerait sur eux l'urbanité dominante (capacitation/empowerment).

\section{Remerciements}

Les auteurs tiennent à adresser leurs vifs remerciements à Jacques Rémy et Fabrice Ripoll pour la pertinence et le caractère toujours constructif de leurs remarques et suggestions.

\section{Bibliographie}

Allen A., Fritzsimmons M., Goodman M., Warner K., 2003. Shifting plates in the agrifood landscape : the tectonics of alternative agrifood initiatives in California, Journal of Rural Studies, vol. 19, n 1, p. 61-75.

Allen A., Guthman J., 2006. From "old school" to "farm-toschool": Neoliberalization from the ground up. Agriculture and Human Values, vol. 23, p. 401-415.

Allirot M., Chevallier M., Lagrange D., Leyssenne C., Zampini C., 2012. Les CCP en Limousin en 2010 : état des lieux, facteurs de réussite et perspectives de développement, Agreste Limousin n ${ }^{\circ} 74$, numéro spécial, 96 p.

Argent N, et al., 2009. Rural amenity and rural change in temperate Australia: implications for development and sustainibility, Revija za geografijo - Journal for Geography, $\mathrm{n}^{\circ}$ 4-2, pages 15-28.

Blanc J., 2012. Construire l'alternative agro-alimentaire : Ressorts sociaux et politiques du déploiement des AMAP en Île-de-France, Norois. Environnement, aménagement, société, 224, p. 21-34.

Bourdieu P., 1979. La distinction : critique sociale du jugement, Éditions de Minuit, Paris, 672 p.

32. Des analyses en cours sur les mêmes données permettent d'ailleurs de constater que les inégalités de revenus entre agriculteurs sont plus importantes au sein des exploitations pratiquant les CCP que dans l'ensemble des exploitations agricoles (Chevallier, 2013b).
Bryson J., Wyckoff W., 2010. Rural gentrification and nature in the Old and New Wests, Journal of Cultural Geography 27 (1), p. 53-75.

Cartier K.C., 1994. Direct marketing of produce: A study of farmers' markets in Jackson, Knoxville, and Memphis, Tennessee, unpublished master's thesis, University of Tennessee, Knoxville, cite dans Detre et al. (2011).

Chevallier M., 2013a. L'hétérogénéité des CCP : un défi pour le soutien institutionnel, Agreste Limousin n 96, 4 p.

Chevallier M., 2013b. Les CCP : une voie rémunératrice mais risquée, Agreste Limousin, sous presse, 4 p.

Chevallier M, Dellier J., Plumecocq G., Richard F., 2014. Dynamiques et structuration des CCP agroalimentaires en Limousin : distance institutionnelle, proximités spatiale et relationnelle, sous presse.

Chiffoleau, Y., 2008. Les CCP de commercialisation en agriculture : diversité et enjeux pour le développement durable, in Maréchal G. (dir.), Les circuits courts alimentaires, Dijon, Educagri Editions, p. 21-30.

Capt D., Chiffoleau Y, Gauche A., 2011. Élaboration d'un référentiel technico-économique dans le domaine des circuits courts de commercialisation, Ministère de l'Agriculture, de la Pêche, de la Ruralité et de l'Aménagement du Territoire, 3 parties, $380 \mathrm{p}$.

Cloke P., Phillips M., Thrift N., 1995. The new middle classes and the social constructs of rural living, in BUTLER T., Savage M. (ed.), Social change and the middle classes, London, UCL Press, p. 220-240.

Cognard F., 2010, «Migrations d'agrément » et nouveaux habitants dans les moyennes montagnes françaises : de la recomposition sociale au développement territorial. L'exemple $d u$ Diois, du Morvan et du Séronais, Thèse de doctorat en géographie, Université Blaise Pascal - Clermont-Ferrand II.

Colletis G., Pecqueur B., 2005. Révélation de ressources spécifiques et coordination située, Économie et Institutions $-\mathrm{n}^{\circ} 6$ et $7-1^{\text {er }}$ et $2^{\mathrm{e}}$ semestres 2005, p. 51-74.

Curry G., Koczberski G., Selwood J., 2001. Cashing Out, Cashing In rural change on the south coast of Western Australia. Australian Geographer Vol. 32 (n 1), p. 109-124.

DARLing E., 2005. The city in the country: wilderness gentrification and the rent gap, Environment and Planning A, vol.37, p. 1015-1032.

Dellier J., Garnier E., Richard F., Attractivité et nouvelles bases économiques des territoires ruraux limousins, Rapport d'étude pour le Conseil Régional du Limousin, 2013, 159 p.

Detre J., Mark T., Mishra A., Adhikari A., 2011 . Linkage Between Direct Marketing and Farm Income: A DoubleHurdle Approach, Agribusiness, Vol. 27 (1), p. 19-33.

Deverre C., Lamine C., 2010. Les systèmes agroalimentaires alternatifs. Une revue de travaux anglophones en sciences sociales, Économie rurale, $\mathrm{n}^{\circ} 317$, p. 57-73.

Dufeu I., Ferrandi J., 2012. Les AMAP ou l'insertion des consommateurs, chemin faisant, dans l'univers de la production agricole de proximité, Actes du Symposium PSDR 3, Les chemins du développement durable, Clermont-Ferrand, 19-21 juin. 
FEAgan R., 2007. The place of food : mapping out the "local" in local food systems, Progress in Human Geography, vol. 31, $n^{\circ} 1$, p. 23-42.

Fournier S., Sirieix L., 2009. Les CCP : perceptions et pratiques des consommateurs du Languedoc-Roussillon, Les Cahiers de l'Observatoire Coxinel, $\mathrm{n}^{\circ} 2$.

Girard A., 2012, Un problème de catégorisation des résidents en amont de la comparaison « autochtones/post-touristes ", in Niels Martin et al., Les migrations d'agrément : du tourisme à l'habiter, L'Harmattan, p. 331-346.

Ghose R., 2004. Big Sky or Big Sprawl? Rural Gentrification and the Changing Cultural Landscape of Missoula, Montana, Urban Geography 25 (6), p. 528-549.

Goodman D., Watts M. (eds.), 1997. Globalising food: agrarian questions and global restructuring. London, routledge.

Govindasamy R., NAyga R., 1996. Characteristics of farmer-toconsumer direct market customers: An overview. Journal of Extension, vol.34, cite dans Detre et al. (2011).

Govindasamy R., Hossain F., Adelaja A., 1999. Income of farmers who use direct marketing. Agricultural and Resource Economics Review, vol.28, p. 76-83.

Henneberry S. R., Whitacre B., Agustini H. N., 2009. An Evaluation of the Economic Impacts of Oklahoma Farmers Markets. Journal of Food Distribution Research 40 (3), p. 64-78.

Hines J. D., 2010. In pursuit of experience: The postindustrial gentrification of the rural American West, Ethnography 11 (2), p. 285-308.

Hines J. D., 2012. The Post-Industrial Regime of Production/ Consumption and the Rural Gentrification of the New West Archipelago, Antipode 44 (1), p. 74-97.

Hinrichs C. C., 2000. Embeddedness and local food systems : notes on two types of direct agricultural market, Journal of Rural Studies, vol. 16, n 3, p. 295-303.

Hoggart K., 1997. The middle classes in rural England, 19711991, Journal of Rural Studies, vol. 13, p. 253-273.

Houdart M., Loudiy S., Gueringer A., 2012. L'adaptation des agriculteurs au contexte périurbain. Norois. Environnement, aménagement, société, $\mathrm{n}^{\circ} 224$, p. 35-48.

LAGARDE V., 2004. Influence du profil du dirigeant sur le type de diversifications en petite entreprise. Application an cas agricole, Thèse en Sciences de Gestion, Faculté de Droit et des Sciences Économiques de Limoges, 420 p. (+ annexes).

Lagarde V., 2006. Le profil du dirigeant comme variable explicative des choix de diversification en agriculture, La Revue Des Sciences De Gestion : Direction Et Gestion, 2006/4-5 (n²20-221), p. 31-41.

Lamine C., Perrot N., 2008. Les AMAP : un nouveau pacte entre producteurs et consommateurs?, Paris, Yves Michel, $163 \mathrm{p}$

LANG T., 2000. Politique alimentaire au XXI ${ }^{\mathrm{e}}$ siècle: à la fois radicale et raisonnable? in Koc M. et al., (dir.), 2000. Armer les villes contre la faim: systèmes alimentaires urbains durables, CRDI. Centre de recherche pour le développement international. Ottawa. Canada, 233 p.
LEYSSENNE C., 2012. Les aides au développement rural pèsent un quart des soutiens publics aux agriculteurs limousins en 2010, Agreste Limousin, ${ }^{\circ}$ 69, 4 p.

LitTle J., 1987. Rural gentrification and the influence of locallevel planning, in Rural planning: policy into action?, Cloke P. Ed., London, Harper \& row, p. 185-199.

Mardden T., Murdoch J., Lowe P., Munton R., Flynn A., 1993. Constructing the countryside, Routledge, London, 232 p.

Mundler P., 2007. Les Associations pour le maintien de l'agriculture paysanne (AMAP) en Rhône-Alpes, entre marché et solidarité, Ruralia, p. 2-20.

Paranthoën J., 2013. Distinction d'une petite bourgeoisie rurale. Un cas d'Amap, Agone n 51, p. 117-130

Pacione M., 1984. Rural Geography, Longman Higher Education.

PARsons D., 1980, Rural gentrification: the influence of rural settlement planning policies. University of Sussex research papers in Geography, $37 \mathrm{p}$.

Perrenoud M., 2008. Les artisans de la «gentrification rurale » : trois manières d'être maçon dans les Hautes-Corbières, Sociétés contemporaines, n 71 (3), p. 95-115.

Perrenoud M., 2012. Artisanat et gentrification rurale en France méridionale, SociologieS. [http://sociologies.revues. $\operatorname{org} / 3991]$.

Phillips M., 1993. Rural gentrification and the processes of class colonization, Journal of Rural Studies 9 (2), p. 123-140.

Phillips M., 2007. Changing class complexions on and in the British countryside, Journal of Rural Studies, vol. 23, p. 283304.

Phillips M., Page S., Saratsi E., Tansey K, Moore K., 2008. Diversity, scale and green landscapes in the gentrification process: Traversing ecological and social science perspectives, Applied Geography 28 (1), p. 54-76.

Pistre P., 2011 , Rural gentrification and ageing: a case study from French countrysides, European Regional Science Association, conference paper, [http://ideas.repec.org/p/wiw/ wiwrsalersal0p 1097.html].

Pistre P., 2012. Renouveaux des campagnes françaises. Evolutions démographiques, dynamiques spatiales et recompositions sociales, Thèse de doctorat en géographie, Paris Diderot (Paris 7), 407 p.

Raymond S., 2005. Des risques de marginalisation aux risques de gentrification : espaces ruraux convoités et inégalement accessibles, In Arlaud et al. (dir.), Rural-Urbain, nowveaux liens, nouvelles frontières, Rennes, PUR, p. 235-249.

Richard F., 2010. La gentrification des « espaces naturels » en Angleterre : après le front écologique, l'occupation?, L'Espace Politique. Revue en ligne de géographie politique et de géopolitique (9).

Richard F., Dellier J., 2011 . Environnements, Migrations et recompositions sociales des campagnes limousines, l'exemple du PNR de Millevaches, rapport d'étude Geolab UMR 6042, Région Limousin, 197 p.

Ripoll F., 2010. L'économie " solidaire » et " relocalisée » comme construction d'un capital social de proximité. Le cas des Associations pour le maintien d'une agriculture paysanne (AMAP). Regards Sociologiques, n 40, p. 59-75. 
Santini F., Gomez y Paloma S. (ed.), 2013. Short Food Supply Chains and Local Food Systems in the EU. A State of Play of their Socio-Economic Characteristics, European Commission, EUR 25911 - Joint Research Centre - Institute for Prospective Technological Studies Luxembourg, Publications Office of the European Union, 154 p.

Sмiтн D., 1998. The revitalisation of the Hebden Bridge District : gentrified Pennine rurality, Phd of Geography, University of Leeds, 358 p.

Smith D.P., Phillips D.A., 2001. Socio-cultural representations of greentrified Pennine rurality, Journal of Rural Studies 17 (4), p. 457-469.

Solana-Solana M., 2010. Rural gentrification in Catalonia, Spain: A case study of migration, social change and conflicts in the Empordanet area, Geoforum 41 (3), p. 508-517.

Stockdale A., 2010. The diverse geographies of rural gentrification in Scotland, Journal of Rural Studies 26 (1), p.31-40.
Sutherland L.A., 2012. Return of the Gentleman Farmer?: Conceptualising Gentrification in UK Agriculture, Journal of Rural Studies 28 (4), p. 568-576.

Transrural Initiatives (TRI), 2006, 2007. Dossier Longue vie aux CCP (1), n³13; (2), n³33.

Van der Ploeg J.D., Renting H., Brunori G., Knickel K., Mannion J., Marsden T, de Roest K, Sevilla-Guzmán E., Ventura F, 2000. Rural development: From practices and policies towards theory, Sociologia ruralis, Vol. 40, $\mathrm{n}^{\circ} 4$, p. $391-408$

Watts D.C.H., Ilbery B., MaYe D., 2005. Making reconnections in agro-food geography : alternative systems of food provision, Progress in Human Geography, vol. 29, $\mathrm{n}^{\circ} 1$, p. 22-40.

Winter M., 2003. Embeddedness, the new food economy and defensive localism, Journal of Rural Studies, vol. 19, $\mathrm{n}^{\circ} 1$, p. 23-32. 